\title{
Histological pattern of Merkel cell carcinoma sentinel lymph node metastasis improves stratification of Stage III patients
}

Jennifer S Koํㄹ Victor G Prieto ${ }^{2}$, Paul J Elson ${ }^{3}$, Ricardo E Vilain ${ }^{4}$, Melissa P Pulitzer ${ }^{5}$, Richard A Scolyer ${ }^{4}$, Jordan P Reynolds ${ }^{1}$, Melissa P Piliang ${ }^{1,6}$, Marc S Ernstoff ${ }^{7}$, Brian R Gastman ${ }^{8}$ and Steven D Billings ${ }^{1}$

${ }^{1}$ Department of Pathology, Cleveland Clinic, Cleveland, Ohio, USA; ${ }^{2}$ Department of Pathology, MD Anderson Cancer Center, Houston, Texas, USA; ${ }^{3}$ Department of Quantitative Health Sciences, Cleveland Clinic, Cleveland, Ohio, USA; ${ }^{4}$ Tissue Pathology and Diagnostic Oncology, Royal Prince Alfred Hospital, Sydney; Melanoma Institute Australia, North Sydney; Sydney Medical School, The University of Sydney, Sydney, New South Wales, Australia; ${ }^{5}$ Department of Pathology, Memorial Sloan-Kettering Cancer Center, New York, New York, USA; ${ }^{6}$ Department of Dermatology, Cleveland Clinic, Cleveland, Ohio, USA; ${ }^{7}$ Department of Hematology Oncology, Melanoma Program, Cleveland Clinic, Cleveland, Ohio, USA and ${ }^{8}$ Department of Plastic Surgery, Cleveland Clinic, Cleveland, Ohio, USA

\begin{abstract}
Sentinel lymph node biopsy is used to stage Merkel cell carcinoma, but its prognostic value has been questioned. Furthermore, predictors of outcome in sentinel lymph node positive Merkel cell carcinoma patients are poorly defined. In breast carcinoma, isolated immunohistochemically positive tumor cells have no impact, but in melanoma they are considered significant. The significance of sentinel lymph node metastasis tumor burden (including isolated tumor cells) and pattern of involvement in Merkel cell carcinoma are unknown. In this study, 64 Merkel cell carcinomas involving sentinel lymph nodes and corresponding immunohistochemical stains were reviewed and clinicopathological predictors of outcome were sought. Five metastatic patterns were identified: (1) sheet-like ( $n=38,59 \%)$; (2) non-solid parafollicular $(n=4,6 \%)$; (3) sinusoidal, $(n=11,17 \%)$; (4) perivascular hilar $(n=1,2 \%)$; and (5) rare scattered parenchymal cells $(n=10,16 \%)$. At the time of follow-up, 30/63 (48\%) patients had died with $21(33 \%)$ attributable to Merkel cell carcinoma. Patients with pattern 1 metastases had poorer overall survival compared with patients with patterns $2-5$ metastases $(P=0.03)$, with $22 / 30(73 \%)$ deaths occurring in pattern 1 patients. Three $(10 \%)$ deaths occurred in patients showing pattern 5 , all of whom were immunosuppressed. Four $(13 \%)$ deaths occurred in pattern 3 patients and $1(3 \%)$ death occurred in a pattern 2 patient. In multivariable analysis, the number of positive sentinel lymph nodes (1 or 2 versus $>2$, $P<0.0001$ ), age ( $<70$ versus $\geq 70, P=0.01$ ), sentinel lymph node metastasis pattern (patterns $2-5$ versus 1 , $P=0.02$ ), and immune status (immunocompetent versus suppressed, $P=0.03$ ) were independent predictors of outcome, and could be used to stratify Stage III patients into three groups with markedly different outcomes. In Merkel cell carcinoma, the pattern of sentinel lymph node involvement provides important prognostic information and utilizing this data with other clinicopathological features facilitates risk stratification of Merkel cell carcinoma patients who may have management implications.
\end{abstract}

Modern Pathology (2016) 29, 122-130; doi:10.1038/modpathol.2015.109; published online 6 November 2015

Merkel cell carcinoma is an aggressive primary cutaneous neuroendocrine carcinoma of increasing

Correspondence: $\mathrm{Dr}$ JS Ko, $\mathrm{MD}, \mathrm{PhD}$ or $\mathrm{Dr}$ SD Billings, $\mathrm{MD}$, Cleveland Clinic, Department of Pathology, 9500 Euclid Avenue L25, Cleveland, Ohio 44195, USA.

E-mail: koj2@ccf.org or billins@ccf.org

Received 12 May 2015; revised 29 July 2015; accepted 30 July 2015; published online 6 November 2015 incidence. ${ }^{1-3}$ Carcinogenesis occurs via viral integration of the retinoblastoma protein sequestering Merkel cell polyomavirus, ${ }^{4,5}$ and ultraviolet radiation-induced mutations, commonly involving the TP53 and Retinoblastoma genes. Both ultraviolet radiation and Merkel cell polyomavirus-induced tumors share clinical features, including aggressive course and propensity for lymphatic spread. 6 Virus negative tumors, have increased mutational burden 
compared with virus-positive cases, and show a relative increase in the percentage of cytokeratin 20 (CK20) negative tumors. ${ }^{7,8}$ Because of the relative rarity of Merkel cell carcinoma compared with melanoma, there are limited data regarding its management. ${ }^{1}$ Histological characteristics in the primary tumor alone do not predict lymph node status. ${ }^{9,10}$ Clinically tumor-involved lymph nodes present at diagnosis portends worse outcome. ${ }^{11-13}$ And now sentinel lymph node biopsy is used for pathological staging, as a guide for further surgical intervention. ${ }^{1}$

Previous studies have shown that immunohistochemistry improves the detection rate of Merkel cell carcinoma in lymph node biopsies. Indeed, Su et al ${ }^{14}$ have reported a $\sim 40 \%$ rate of immunohistochemistry positive Merkel cells in hematoxylin and eosin (H\&E) negative sentinel lymph nodes. In a related study by Allen et al, ${ }^{15}$ investigating sentinel lymph node biopsies in a series of 26 patients, $40 \%$ ( 2 out of 5) of Merkel cell carcinoma positive sentinel lymph nodes were negative on $\mathrm{H} \& \mathrm{E}$ analysis. The aim of these studies, however, was to evaluate the utility and not the clinical meaning of immunohistochemistry in Merkel cell carcinoma sentinel lymph node biopsies; and reports evaluating the clinical significance of isolated tumor cells in sentinel lymph nodes are lacking.

In this study, we sought: (1) to characterize the patterns of involvement of sentinel lymph node biopsies by Merkel cell carcinoma, both $\mathrm{H} \& \mathrm{E}$ evident and immunohistochemistry-dependent, in a large cohort of cases; (2) to correlate this pattern and other clinicopathological characteristics with outcome; and (3) to determine the significance metastatic tumor detected only by immunohistochemistry.

\section{Materials and methods}

The study was approved by appropriate institutional review boards. The pathology archives from the involved institutions were searched for sentinel lymph node biopsies that were positive for Merkel cell carcinoma. The sentinel lymph nodes and any corresponding immunostains were reviewed for pattern of metastasis in 64 patients with adequate follow-up. Immunohistochemical stains typically included cytokeratin 20 (CK20) and/or pan-cytokeratin stains. We observed that lymph node involvement could generally be categorized into five patterns, and these patterns were arbitrarily designated by numbers in the order that they were discovered until patterns became redundant (see results section). The five patterns of sentinel lymph node biopsy involvement were: pattern 1-sheet-like (with and without extracapsular extension); 2-parafollicular, non-solid; 3-sinusoidal; 4-perivascular hilar; and 5-single scattered cells. It was further noted if the tumor cells were identified by immunohistochemistry only. Follow-up, demographics, and clinical data, including patient immune status at the time of sentinel lymph node biopsy, were obtained from patients' medical records.

We also evaluated the effect of immunosuppression on outcome, as Merkel cell carcinoma can be virally induced and has been shown to be immunogenic. We investigated whether patients who were immunosuppressed before and at the time of diagnosis had comparable outcome to immunocompetent patients, and whether pattern of sentinel lymph node involvement carried the same prognostic significance in immunocompetent and immunosuppressed patients. For the purposes of this study, immunosuppression was defined as having a comorbidity associated with immune suppression (eg, hematological malignancy) or concurrent treatment with immunosuppressive medications. The primary outcome of interest was overall survival which was measured from the date of diagnosis to the date of death from any cause or last follow-up. Categorical factors were summarized as frequency counts and percentages and measured factors as medians and ranges. The Kaplan-Meier method was used to summarize overall survival. Fisher's exact test and chi-square tests were used to assess associations between clinical factors and sentinel lymph node pattern. The logrank test and proportional hazards models were used to assess univariable and multivariable associations with overall survival. Data were analyzed using SAS version 9.4 (SAS Institute, Cary, NC, USA) and StatXact 10 (Cytel, Cambridge, MA, USA).

\section{Results}

\section{Patient Characteristics}

Table 1 incorporates some of the clinical characteristics of the patients in this cohort along with univariate survival analysis data discussed below. The most common primary tumor site was the extremities $(29 / 64,46 \%)$ including five tumors originating in the hand. Head and neck was the next most common site affected $(20 / 64,31 \%)$. These findings are compatible with the mechanistic role that ultraviolet radiation has in Merkel cell carcinogenesis. Immunosuppressed patients $(14 / 61$ (data missing for three patients)) comprised $23 \%$ of the patient cohort, which is certainly an overrepresentation compared with the general public, and is consistent with the role that Merkel cell polyomavirus has in tumorigenesis, as well as the role of immunosurveillance in the elimination of occult cancers. ${ }^{16,17}$ Overall, 28 out of 62 patients with follow-up had tumor recurrence (45\%, not shown). Survival information was available for 63 patients. Overall, 30 (48\%) patients have died; and $21(70 \%)$ of the deaths were directly attributable 
Table 1 Clinical parameters and overall survival- univariable analysis

\begin{tabular}{|c|c|c|c|c|}
\hline Factor & $\mathrm{N}$ & $\begin{array}{c}\text { 2-Year } \\
\text { survival } \pm \text { s.e.m. }\end{array}$ & Median & $\mathrm{P}^{\mathrm{a}}$ \\
\hline \multicolumn{5}{|l|}{ Gender } \\
\hline Male & $41(65 \%)$ & $63 \% \pm 8 \%$ & 44 & \\
\hline Female & $24(35 \%)$ & $71 \% \pm 10 \%$ & 66 & 0.41 \\
\hline \multicolumn{5}{|c|}{ Age at Diagnosis } \\
\hline Total & (51-90 years) & $77 \% \pm 8 \%$ & 70 years & \\
\hline$<70$ & 31 & & N/A & \\
\hline$>70$ & 32 & $56 \% \pm 9 \%$ & 34 & 0.004 \\
\hline \multicolumn{5}{|l|}{ Location } \\
\hline Head/neck & $20(31 \%)$ & $48 \% \pm 12 \%$ & 23 & \\
\hline Extremities & $29(46 \%)$ & $76 \%+9 \%$ & 107 & \\
\hline Other & $15(23 \%)$ & $70 \%+13 \%$ & 107 & 0.09 \\
\hline \multicolumn{5}{|c|}{ No. positive sentinel lymph nodes } \\
\hline 1 & 47 & $71 \% \pm 7 \%$ & 73 & \\
\hline 2 & 11 & $63 \% \pm 18 \%$ & 42 & \\
\hline$>2$ & 4 & $0 \%$ & 4 & $<0.0001^{\mathrm{b}}$ \\
\hline \multicolumn{5}{|c|}{ Sentinel lymph node pattern } \\
\hline 1 & 38 & $61 \% \pm 9 \%$ & 35 & \\
\hline 2 & 4 & $100 \%$ & N/A & \\
\hline 3 & 11 & $73 \%+13 \%$ & N/A & \\
\hline 4 & 1 & $100 \%$ & N/A & \\
\hline 5 & 10 & $53 \% \pm 20 \%$ & N/A & 0.28 \\
\hline 1 & 38 & $61 \% \pm 9 \%$ & 35 & \\
\hline $2-5$ & 26 & $73 \%+9 \%$ & N/A & 0.03 \\
\hline \multicolumn{5}{|c|}{ Extracapsular extension (pattern 1) } \\
\hline No & 10 & $70 \% \pm 14 \%$ & 107 & \\
\hline Yes & 15 & $56 \% \pm 14 \%$ & 26 & 0.27 \\
\hline \multicolumn{5}{|c|}{ Immunosuppressed } \\
\hline No & 47 & $78 \% \pm 7 \%$ & 73 & \\
\hline Yes & $14(23 \%)$ & $40 \% \pm 14 \%$ & 19 & 0.12 \\
\hline
\end{tabular}

Abbreviation: N/A, not applicable.

${ }^{\text {a }}$ Logrank test unless otherwise noted.

$\mathrm{b}_{\text {Logrank test for trend. }}$

to Merkel cell carcinoma (not shown). Estimated overall median survival was 46.0 months (95\% confidence interval 26.0-107.0 months, not shown). Median (range) follow-up for the 33 patients alive at the time of this analysis was 34.0 (3.0-160.0, not shown) months.

\section{Patterns of Sentinel Lymph Node Biopsy Involvement by Merkel Cell Carcinoma}

All of the cases of Merkel cell carcinoma involving a sentinel lymph node could be grouped into one of five patterns (Figure 1). The first pattern seen (pattern 1-sheet-like) was characterized by a solid, sheet-like tumor nodule involving variable amounts of lymph node parenchyma (Figure 1a). This pattern was typically easily identified on $\mathrm{H} \& \mathrm{E}$ stained sections and did not necessitate immunohistochemistry for diagnosis, but only for confirmation of tumor type. The second pattern seen (pattern 2-parafollicular, non-solid) was characterized by non-solid, dispersed cells clustered in the parafollicular lymph node cortex (Figure 1b). Pattern 3 -sinusoidal showed variable numbers of isolated cells sprinkled within the subcapsular and draining lymph node sinuses (Figure 1c). Pattern 4 (perivascular hilar) was quite rare, but showed tumor cells clustered around the larger vessels in the lymph node hilum (Figure 1d). Pattern 5 (single scattered cells) showed rare, isolated parenchymal tumor cells (Figure 1e). Patterns 2-4 often required immunohistochemistry for visualization, while pattern 5 always required immunohistochemical stains to detect metastatic tumor. Patterns $2-5$ typically had $<200$ tumor cells in the involved lymph node.

\section{Relative Prevalence of Tumor Patterns in Sentinel Lymph Node Biopsies and Associations with Clinical Factors}

The relative prevalence of the different patterns of sentinel lymph node involvement by Merkel cell carcinoma is shown in Figure 2. A sheet-like, solid pattern (pattern 1) of variably-sized lymph node parenchymal deposits of Merkel cell carcinoma was most commonly seen in sentinel lymph node biopsies $(38 / 64,59 \%)$. The next most common pattern seen was the sinusoidal, pattern 3, which involved 11/64 cases (17\%). Next most common, was the pattern of finding rare isolated CK20-positive cells in the lymph node parenchyma (pattern 5, 10/64 cases, 16\%). CK20-positive cells were not seen in control cases of sentinel lymph node biopsies from patients being treated for melanoma (not shown). Pattern 2 was seen in 4 out of 64 cases $(6 \%)$ and pattern 4 in 1 out of 64 cases $(2 \%)$. There was no indication that sentinel lymph node pattern was associated with gender, age, location of the primary, or immune status (all $P \geq 0.38$ ). Not surprisingly, extracapsular extension was associated with pattern 1 , such that among 44 patients with data, 15 (34\%) had extracapsular extension, and all 15 had sentinel lymph node pattern 1 $(P<0.0001)$. In addition, pattern 1 patients tended to have more than one positive sentinel lymph node more frequently than patients with patterns $2-5$ (32\%, $12 / 37$ versus $8 \%, 2 / 26$, respectively; $P=0.05$ ).

\section{Patient Outcomes Vary Significantly with Pattern of Sentinel Lymph Node Biopsy Involvement, Number of Involved Lymph Nodes and Clinical Factors}

The association of various clinicopathological factors with survival in Merkel cell carcinoma patients is summarized in Table 1. There was no indication that survival differed in patients with sentinel lymph node patterns 2 through $5(P=0.28)$; however patients with pattern 1 tended to have worse overall survival than patients with these other 

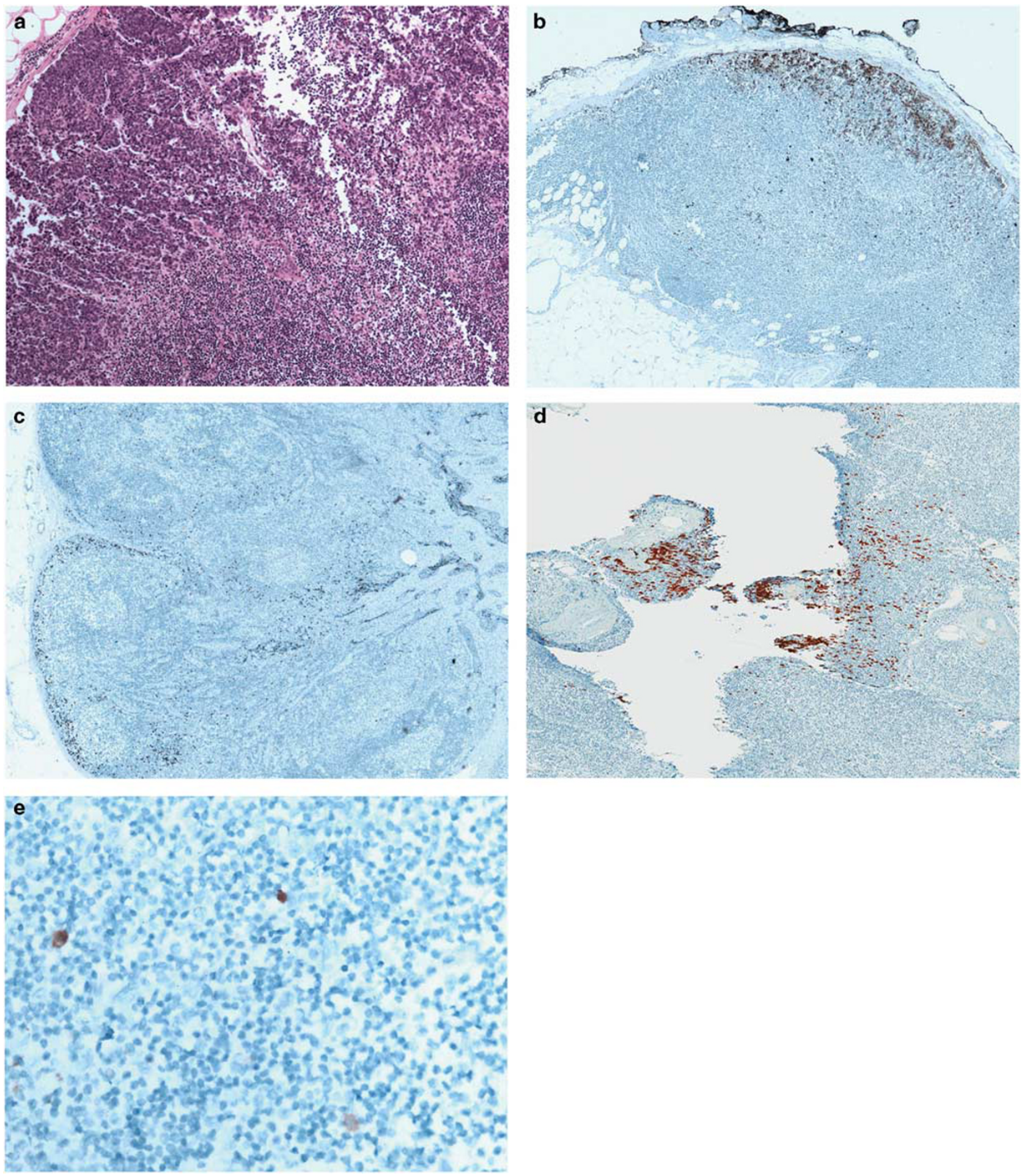

Figure 1 Patterns of lymph node involvement by Merkel cell carcinoma. (a) Pattern 1 was characterized by a solid, sheet-like proliferation of metastatic tumor. Immunohistochemistry was not needed to identify metastatic tumor in these cases (H\&E). (b) Pattern 2 was characterized by a non-solid proliferation of tumor cells in the parafollicular lymph node cortex (CK20 immunohistochemical stain). (c) Pattern 3 was characterized by variable numbers of tumor cells in the subcapsular sinus (CK20 immunohistochemical stain). (d) Pattern 4 was characterized by clusters of tumor cells around larger vessels in the lymph node hilum. This was the least common pattern of metastasis (CK20 immunohistochemical stain). (e) Pattern 5 was characterized by isolated rare tumor cells in the lymph node parenchyma. This pattern of metastasis was only detected by immunohistochemistry (CK20 immunohistochemical stain). CK20, cytokeratin 20; H\&E, hematoxylin and eosin. 
patterns $(P=0.03$, Figure 3a). Twenty-two out of thirty $(73 \%)$ deaths occurred in pattern 1 patients. Three deaths occurred in patients showing pattern 5, and all three were immunosuppressed. Four deaths occurred in pattern 3 patients and 1 death occurred in a pattern 2 patient. Other factors associated with poorer overall survival were increased number of positive sentinel lymph nodes $(P<0.0001$, Figure $3 \mathrm{~b})$ and older age at diagnosis $(\geq 70, P=0.004$, Figure 3c). There was also some suggestion that patients with primary tumors on the
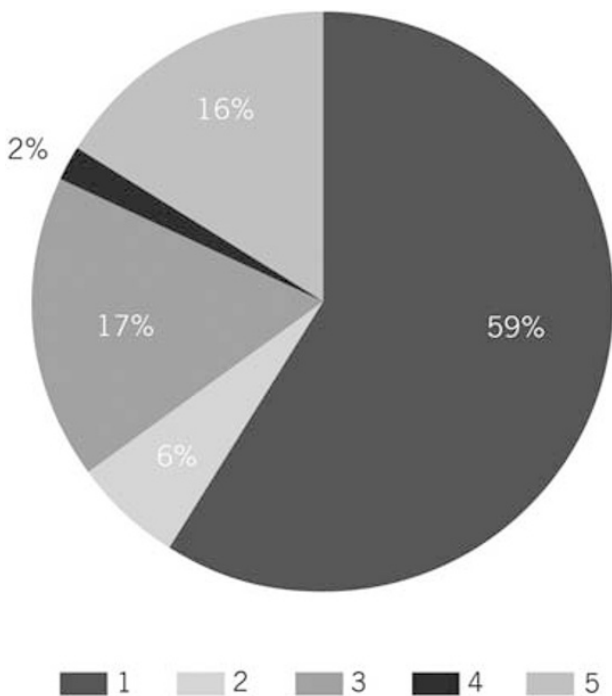

Figure 2 Distribution of patterns of metastasis. Pattern 1 was the most common pattern of lymph node involvement. Patterns 3 and 5 were present in roughly equivalent percentages. Patterns 2 and 4 were relatively rare. head had a worse prognosis than patients with tumors in other areas $(P=0.09$, not shown).

Because Merkel cell carcinoma can be virally induced and has been shown to be immunogenic, we investigated whether patients who were immunosuppressed had comparable outcome to immunocompetent patients, and whether pattern of sentinel lymph node involvement carried the same prognostic significance in immunocompetent and immunosuppressed patients. Immunosuppressive comorbidities included chronic lymphocytic leukemia, diffuse large B-cell lymphoma, myelodysplastic syndrome, chronic myelogenous leukemia, plasma cell neoplasm, status-post liver or heart transplantation, myasthenia gravis, inclusion body myositis and autoimmune thrombocytopenic purpura on immunosuppressive medication. Overall, immunosuppressed patients had poorer survival compared with immunocompetent patients, although in univariate analysis the difference was not statistically significant $(P=0.12$, not shown). Similarly, pattern 1 patients had poorer survival in immunosuppressed and immunocompetent patients, respectively; however, the differences were not statistically significant when the two groups were separated $(P=0.19$ and 0.09 , respectively, not shown), possibly due to the reduction in cohort size resulting from this division.

In multivariable analysis that initially considered the above factors and gender, the number of positive sentinel lymph nodes present (1 or 2 versus $>2$, $P<0.0001$ ), age ( $<70$ versus $\geq 70, P=0.01$ ), sentinel lymph node pattern (patterns $2-5$ versus $1, P=0.02$ ), and immune status (immunocompetent versus suppressed, $P=0.03$ ) were seen to be independent predictors of outcome (Table 2). The resulting model a

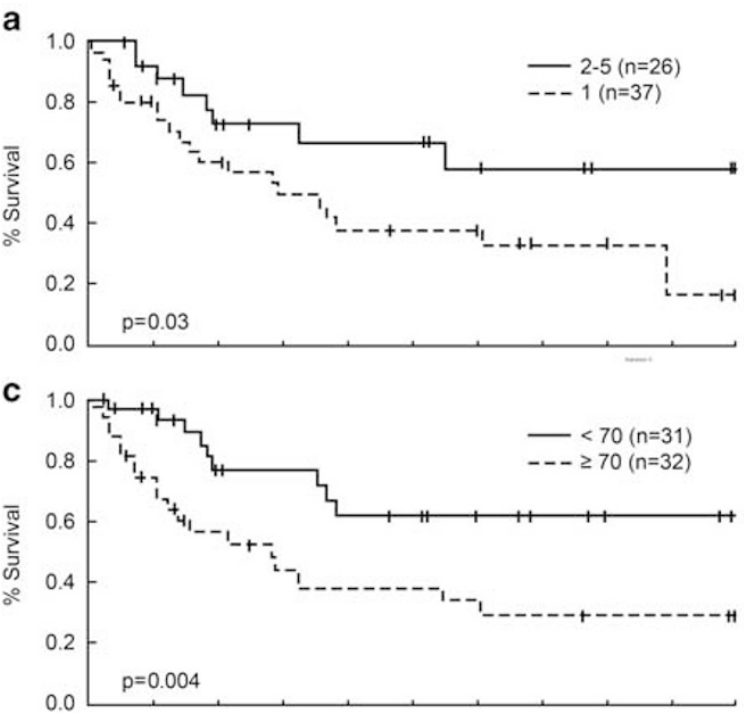

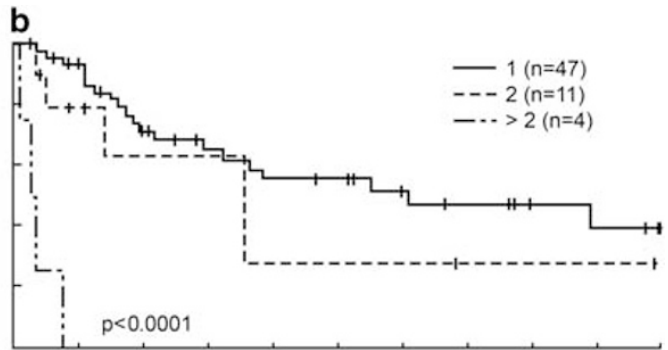$$
\text { . }
$$

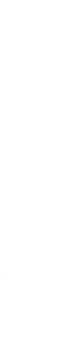

Figure 3 Clinicopathological factors related to overall survival. (a) Pattern 1 was associated with a worse outcome compared with the combined patterns 2-4. (b) Involvement of more than one sentinel lymph node biopsy was associated with a significantly worse overall survival. (c) Age greater than 70 years was associated with a worse overall survival. 
Table 2 Multivariable analysis

\begin{tabular}{|c|c|c|c|}
\hline Factor & $\begin{array}{c}\text { Regression } \\
\text { coefficient } \pm \text { s.e.m. }\end{array}$ & Hazard ratio $(95 \% C I)^{\mathrm{a}}$ & $\mathrm{P}^{\mathrm{b}}$ \\
\hline No. positive SLN ( 1 or 2 vs $>2$ ) & $3.50 \pm 0.82$ & $33.16(6.69-164.35)$ & $<0.0001$ \\
\hline Age at diagnosis $(<70 v s \geq 70)$ & $1.09 \pm 0.43$ & $2.98(1.27-6.98)$ & 0.01 \\
\hline SLN pattern (2-5 vs 1$)$ & $1.12 \pm 0.46$ & $3.07(1.23-7.62)$ & 0.02 \\
\hline Immune suppression (no vs yes) & $0.98 \pm 0.46$ & $2.66(1.08-6.58)$ & 0.03 \\
\hline
\end{tabular}

Abbreviations: CI, confidence interval; No., number; SLN, sentinel lymph node.

${ }^{\mathrm{a}}$ The first feature listed serves as the control; hazard ration $=\mathrm{e}^{\text {regression coefficient }}$

${ }^{b}$ Wald test.

Table 3 Prognostic groups based on multivariable analysis

\begin{tabular}{lccrr}
\hline Prognostic groups & No. 'points & N & 2-Year survival \pm s.e.m. & Median \\
\hline Favorable & 0 & $12(20 \%)$ & $90 \% \pm 9 \%$ & N/A \\
Intermediate & $1-2$ & $41(67 \%)$ & $73 \% \pm 8 \%$ & 66.0 \\
Unfavorable & $3-6$ & $8(13 \%)$ & $0 \%$ & 4.0 \\
\hline
\end{tabular}

Abbreviation: No., number.

${ }^{a}$ Age $\geq 70$, LN pattern 1 , and immunosuppression each count as 1 'point'; $>2$ positive SNL counts as 3 .

b Logrank test for trend.

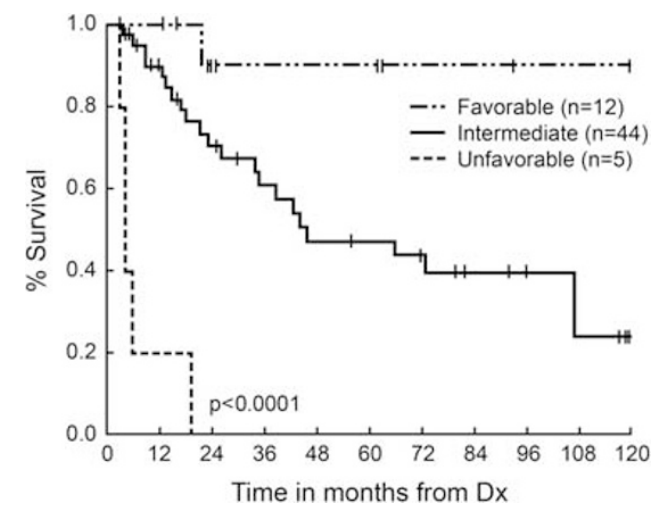

Figure 4 The patients could be stratified into three distinct groups based on the scoring system described in the Results section: (1) favorable (0 points), (2) intermediate (1-2 points), and (3) unfavorable ( $>2$ points). There was clear survival difference between all three groups.

can be simplified by using a scoring system that assigns 'points' (weights) to each factor that are proportional to the corresponding regression coefficients; and then sums the total number of points present. The scores in turn can be combined to form prognostic groups. That is, counting age $\geq 70$, LN pattern 1 , and immune suppression as one 'point' and $>2$ positive sentinel lymph nodes as 3 'points', three prognostic groups can be defined:

1. Favorable-patients with no poor features present (0 points), ie, 1 or 2 positive sentinel lymph nodes, age $<70$, sentinel lymph node pattern $2-5$, immunocompetent; $20 \%$ of patients, median overall survival N/A; 2-year survival $90 \% \pm 9 \%$;

2. Intermediate-patients with 1-2 points; $67 \%$ of patients median and 2-year survival 66.0 months and $73 \% \pm 8 \%$, respectively; and

3. Unfavorable- $>2$ points; $13 \%$ of patients; all patients died within 19 months of diagnosis (Table 3 and Figure 4).

\section{Discussion}

The advent of sentinel lymph node biopsy for pathological regional lymph node sampling has transformed the management of many tumor types, including melanoma, breast carcinoma and Merkel cell carcinoma; ${ }^{18}$ allowing for more accurate lymph node analysis due to increased lymph node serial sectioning, and immunohistochemical staining to identify single tumor cells and small tumor cell clusters. ${ }^{14,15,19}$ In melanoma, immunohistochemistry, and the practice of designating varying degrees of sentinel lymph node involvement as equally positive from a management perspective, has mostly endured. ${ }^{20-22}$ To the contrary, in breast carcinoma, micrometastatic disease in the sentinel lymph nodes, detected with immunohistochemistry alone, does not bear prognostic significance. ${ }^{23,24}$

Regional lymph node evaluation in Merkel cell carcinoma is recommended because of studies showing an orderly cascade of metastasis, often initially involving the draining lymph node basin; ${ }^{11}$ and sentinel lymph node biopsy has become the 
preferred method of evaluation. ${ }^{25,26}$ The significance of infrequent, scattered, dyshesive tumor cells in sentinel lymph node biopsies from Merkel carcinoma is unknown. Clinical tumor positivity in regional lymph nodes has long been known to correlate with worse outcome (5-year survival rate of $<50 \%),{ }^{27}$ and studies by Allen et al ${ }^{13}$ support the prognostic value of pathological lymph node assessment, with clinically lymph node negative patients surviving at $75 \%$ at 5 -years, while pathologically lymph node negative patients survived at $97 \%$ at 5 -years $(P=0.009)$, with $\sim 32 \%$ of clinically node negative patients showing pathologically positive lymph nodes. ${ }^{13,28,29}$ Nevertheless, studies by Allen et $a l^{13}$ and Gupta et $a l^{29}$ give no mention of immunohistochemistry, and thus it is assumed that this was not (at least routinely) used in lymph node evaluation. Meanwhile, studies by Shibayama et al used immununohistochemical staining in a portion of their sentinel node biopsies. In contrast to studies sited earlier in this paper, the rate of positive diagnosis with $(n=269)$ or without $(n=134)$ antiCK 20 antibody usage did not significantly differ (32 vs 31\%, respectively). Furthermore, the use of immunohistochemical staining to diagnose micrometastasis with anti-CK20 antibody did not affect the false-negative rate (regional nodal recurrence in a basin with a previously negative sentinel lymph node). ${ }^{28}$ As such, the prognostic value of immunohistochemically detected carcinoma in the sentinel lymph node biopsies of Merkel cell patients remains ambiguous. ${ }^{30-34}$

Although the use of cytokeratin immunohistochemical staining increases the rate of Merkel cell carcinoma detection in $\mathrm{H} \& \mathrm{E}$ negative lymph nodes, ${ }^{14,15}$ studies investigating the prognostic significance of metastatic tumor detected only by immunohistochemistry are lacking. The current study evaluated sentinel lymph node biopsies which were positive for Merkel cell carcinoma, either on H\&E sections or by immunohistochemical staining; and categorized the histological appearance of tumor involvement in an attempt to understand the significance, if any, of varying patterns of disease.

We have found that the most common pattern of sentinel lymph node involvement, seen in $59 \%$ of patients, is characterized by a diffuse, sheet-like solid growth pattern of varying sizes, which involves the lymph node parenchyma with or without extracapsular extension, corresponding to what we designated pattern 1 . The next most common pattern was characterized by scattered, dyshesive tumor cells of variable number, located in the subcapsular sinus, the draining sinuses, or both, designated pattern 3 and occurring in $17 \%$ of patients. Pattern 5, which was characterized by rare scattered parenchymal cells was the next most common, present in 16\%. Patterns 2 (non-solid, parafollicular) and 4 (tight perivascular hilar) were quite rare.

Our results show that the pattern of sentinel lymph node biopsy involvement by tumor was statistically correlated to patient outcome, such that patients with pattern 1 disease showed decreased survival compared to patterns $2-5$. We suggest that patterns 2-5 be considered equivalent and be considered to represent a pattern of low tumor burden in sentinel lymph node biopsies. There was no significant difference in outcome between the various immunohistochemical staining-dependent pattern groups indicating that when CK20+ tumor cells number less than 200 or so, their distribution may not be meaningful, with a caveat for those rare cases with multiple non-pattern 1 sentinel lymph nodes involved. This trend was apparent in both immunocompetent patients as well as immunosuppressed patients; however, the number of cases evaluated was small. Overall, immunosuppressed patients had a decreased survival time compared to immunocompetent patients in multivariate analysis. Perhaps highlighting the role of immunosuppression further is the fact that all three patients with pattern 5 sentinel lymph node involvement who later died of disease were immunosuppressed.

The rate of death of disease in our cohort was 33\%, which is lower than what is reported for stage III patients. This may be due to limited follow-up on some patients, and the inclusion of patients with pattern 5 as Stage III. Previous studies aimed at testing the utility of particular immunohistochemical stains to detect occult Merkel cell carcinoma tumor in sentinel lymph node biopsies, also reported an incidentally excellent outcome in patients with disease detected only by immunohistochemistry. ${ }^{14,15}$

Although sentinel lymph node biopsy pattern within this cohort of patients significantly predicted outcome, additional factors could further help stratify this group. As such, when number of sentinel lymph nodes positive, age, and immune status were also considered, patients with excellent prognosis ( $20 \%$ of patients), despite Stage III designation, could be identified. Likewise, patients with unusually aggressive disease course (13\% of patients) were isolated. Interestingly, upon review of the data, 14 patients had greater than 1 sentinel lymph node involved, and 2/14 of these patients did not have pattern 1 involvement in any of the affected lymph nodes (1/2 was pattern 3 in three lymph nodes, and $1 / 2$ was pattern 5 in two lymph nodes). Four out of these 14 patients had greater than 2 positive sentinel lymph nodes, and of these four patients, 1 patient had non-pattern 1 involvement (three lymph nodes with pattern 3). Out of these two patients with multiple non-pattern 1 lymph nodes involved, one died of disease despite being non-pattern 1. This patient was over the age of 70 and the tumor was from the hand. Hence, while having at least three sentinel lymph nodes with tumor (four total patients) was most strongly correlated with poor outcome, this was a relatively rare occurrence that was not entirely restricted to pattern 1 patients. 
This is the first study to investigate the meaning of various patterns of sentinel lymph node involvement by Merkel cell carcinoma, including the meaning of disease identified with immunohistochemistry alone. Our findings suggest improved survival in patients with metastatic tumor involving sentinel lymph nodes detected only by immunohistochemistry, and raise the question of whether these patients deserve separate classification and different management, analogous to breast carcinoma. Further investigation with an expanded cohort size and follow-up time is warranted.

\section{Disclosure/conflict of interest}

The authors declare no conflict of interest.

\section{References}

1 Moshiri AS, Nghiem P. Milestones in the staging, classification, and biology of Merkel cell carcinoma. J Natl Compr Canc Netw 2014;12:1255-1262.

2 Lemos B, Nghiem P. Merkel cell carcinoma: more deaths but still no pathway to blame. J Invest Dermatol 2007;127:2100-2103.

3 Heath M, Jaimes N, Lemos B et al. Clinical characteristics of Merkel cell carcinoma at diagnosis in 195 patients: the AEIOU features. J Am Acad Dermatol 2008;58:375-381.

4 Feng H, Shuda M, Chang Y et al. Clonal integration of a polyomavirus in human Merkel cell carcinoma. Science 2008;319:1096-1100.

5 Moore PS, Chang Y. Why do viruses cause cancer? Highlights of the first century of human tumour virology. Nat Rev Cancer 2010;10:878-889.

6 Sahi H, Savola S, Sihto H et al. RB1 gene in Merkel cell carcinoma: hypermethylation in all tumors and concurrent heterozygous deletions in the polyomavirusnegative subgroup. APMIS 2014;122:1157-1166.

7 Sihto H, Kukko $\mathrm{H}$, Koljonen $\mathrm{V}$ et al. Merkel cell polyomavirus infection, large $\mathrm{T}$ angtigen, retinoblastoma protein and outcome in Merkel cell carcinoma. Clin Cancer Res 2011;17:4806-4813.

8 Miner AG, Patel RM, Wilson DA et al. Cytokeratin 20-negative Merkel cell carcinoma is infrequently associated with the Merkel cell polyomavirus. Mod Pathol 2015;28:498-504.

9 Tseng J, Dhungel B, Mills JK et al. Merkel cell carcinoma: what makes a difference? Am J Surg 2015;209:342-346.

10 Schwartz JL, Griffith KA, Lowe L et al. Features predicting sentinel lymph node positivity in Merkel cell carcinoma. J Clin Oncol 2011;29:1036-1041.

11 Yiengpruksawan A, Coit DG, Thaler HT et al. Merkel cell carcinoma. Prognosis and management. Arch Surg 1991;126:1514-1519.

12 Allen PJ, Zhang ZF, Coit DG. Surgical management of Merkel cell carcinoma. Ann Surg 1999;229:97-105.

13 Allen PJ, Bowne WB, Jaques DP et al. Merkel cell carcinoma: prognosis and treatment of patients from a single institution. J Clin Oncol 2005;23:2300-2309.

$14 \mathrm{Su} \mathrm{LD}$, Lowe L, Bradford CR et al. Immunostaining for cytokeratin 20 improves detection of micrometastatic
Merkel cell carcinoma in sentinel lymph nodes. J Am Acad Dermatol 2002;46:661-666.

15 Allen PJ, Busam K, Hill AD et al. Immunohistochemical analysis of sentinel lymph nodes from patients with Merkel cell carcinoma. Cancer 2001;92:1650-1655.

16 Stakaitytė G, Wood JJ, Knight LM et al. Merkel cell polyomavirus: molecular insights into the most recently discovered human tumour virus. Cancers (Basel) 2014;6:1267-1297.

17 Schreiber RD, Old LJ, Smyth MJ. Cancer immunoediting: integrating immunity's roles in cancer suppression and promotion. Science 2011;331:1565-1570.

18 Tseng J, Dhungel B, Mills JK et al. Merkel cell carcinoma: what makes a difference? Am J Surg 2015;209:342-346.

19 Tsuda H. Histological examination of sentinel lymph nodes: significance of macrometastasis, micrometastasis, and isolated tumor cells. Breast Cancer 2015;22:221-229.

20 Balch CM, Gershenwald JE, Soong SJ et al. Multivariate analysis of prognostic factors among 2,313 patients with stage III melanoma: comparison of nodal micrometastases versus macrometastases. J Clin Oncol 2010;28:2452-2459.

21 Murali R, DeSilva C, McCarthy SW et al. Sentinel lymph nodes containing very small $<0.1 \mathrm{~mm}$ ) deposits of metastatic melanoma cannot be safely regarded as tumor-negative. Ann Surg Oncol 2012;19:1089-1099.

22 Morton DL, Thompson JF, Cochran AJ et al. MSLT Group. Final trial report of sentinel-node biopsy versus nodal observation in melanoma. $N$ Engl J Med 2014;370:599-609.

23 Giuliano AE, Hawes D, Ballman KV et al. Association of occult metastases in sentinel lymph nodes and bone marrow with survival among women with early-stage invasive breast cancer. JAMA 2011;306:385-393.

24 Gatzemeier W, Mann GB. Which sentinel lymph-node (SLN) positive breast cancer patient needs an axillary lymph-node dissection (ALND)-ACOSOG Z0011 results and beyond. Breast 2013;22:211-216.

25 Sadeghi R, Adinehpoor Z, Maleki M et al. Prognostic significance of sentinel lymph node mapping in Merkel cell carcinoma: systematic review and meta-analysis of prognostic studies. Biomed Res Int 2014;2014:489536.

26 Grotz TE, Joseph RW, Pockaj BA et al. Negative sentinel lymph node biopsy in merkel cell carcinoma is associated with a low risk of same-nodal-basin recurrences. Ann Surg Oncol 2015;22:4060-4066.

27 Edge SB, Compton CC. The American Joint Committee on Cancer: the 7th Edition of the AJCC Cancer Staging Manual and the Future of TNM. Ann Surg Oncol 2010;17:1471-1474.

28 Shibayama Y, Imafuku S, Takahashi A et al. Role of sentinel lymph node biopsy in patients with Merkel cell carcinoma: statistical analysis of 403 reported cases. Int J Clin Oncol 2015;20:188-193.

29 Gupta SG, Wang LC, Penas PF et al. Sentinel lymph node biopsy for evaluation and treatment of patients with Merkel cell carcinoma: the Dana-Farber experience and meta-analysis of the literature. Arch Dermatol 2006;142:685-690.

30 Lemos BD, Storer BE, Iyer JG et al. Pathologic nodal evaluation improves prognostic accuracy in Merkel cell carcinoma: analysis of 5823 cases as the basis of the first consensus staging system. J Am Acad Dermatol 2010;63:751-761. 
31 Mehrany K, Otley CC, Weenig RH et al. A meta-analysis of the prognostic significance of sentinel lymph node status in Merkel cell carcinoma. Dermatol Surg 2010;28:113-117.

32 Maza S, Trefzer U, Hofmann M et al. Impact of sentinel lymph node biopsy in patients with Merkel cell carcinoma: results of a prospective study and review of the literature. Eur J Nucl Med Mol Imaging 2006;33: $433-440$.
33 Fields RC, Busam KJ, Chou JF et al. Recurrence and survival in patients undergoing sentinel lymph node biopsy for Markel cell carcinoma: analysis of 153 patients from a single institution. Ann Surg Oncol 2011;18:2529-253.

34 Warner RE, Quinn MJ, Hruby G et al. Management of Merkel cell carcinoma: the roles of lymphoscintigraphy, sentinel lymph node biopsy and adjuvant radiotherapy. Ann Surg Oncol 2008;15:2509-2518. 